\title{
High-precision excited state lifetime measurements in rare earth nuclei using $\operatorname{LaBr}_{3}(\mathrm{Ce})$ detectors
}

\author{
E. Williams ${ }^{1, a}$, N. Cooper ${ }^{1}$, M. Bonett-Matiz ${ }^{1}$, V. Werner ${ }^{1}$, J.-M. Régis ${ }^{2}$, M. Rudigier $^{2}$, T. Ahn ${ }^{1}$, V. Anagnostatou ${ }^{1,3}$, Z. $^{1}$ \\ Berant $^{1,4}$, M. Bunce ${ }^{1,3}$, M. Elvers ${ }^{1,2}$, A. Heinz ${ }^{1}$, G. Ilie ${ }^{1}$, J. Jolie ${ }^{2}$, D. Radeck ${ }^{1,2}$, D. Savran ${ }^{1,5}$, and M. Smith ${ }^{1}$ \\ 1 Wright Nuclear Structure Laboratory, Yale University, P.O. Box 208120, New Haven, CT 06520, USA \\ 2 Institut für Kernphysik, Universität zu Köln, Zülpicher Str. 77, 50937 Köln, Germany \\ 3 University of Surrey, Guildford, Surrey, GU2 7XH, United Kingdom \\ 4 Nuclear Research Center Negev, Beer-Sheva 84190, Israel \\ 5 Institut für Kernphysik, Technische Universität Darmstadt, Schlossgartenstr. 9, 64289 Darmstadt, Germany
}

\begin{abstract}
To study how collective nuclear structure evolves towards mid-shell and test next-generation $\mathrm{LaBr}_{3}(\mathrm{Ce})$ scintillation detectors, measurements of the lifetimes of $2_{1}^{+}$states in ${ }^{168} \mathrm{Hf}$ and ${ }^{174} \mathrm{~W}$ were conducted at the Wright Nuclear Structure Laboratory. Preliminary results indicate that the excellent time and energy resolution of $\mathrm{LaBr}_{3}$ detectors make them well suited to fast timing measurements, allowing for improved background subtraction and peak resolution in comparison to $\mathrm{BaF}_{2}$ detectors. Preliminary analysis shows an order of magnitude reduction in the statistical error of the $2^{+}$lifetimes in comparison to literature values for both nuclei. In the case of ${ }^{174} \mathrm{~W}$, a substantial reduction of the observed lifetime hints at the possibility of new physics in the region.
\end{abstract}

\section{Introduction}

High-precision measurements of electromagnetic transition strengths are one of the key observables required for studying nuclear structure phenomena. Transition strengths provide model-independent information on the evolution of nuclear collective excitations as a function of nucleon number [1,2] and help illuminate the location and possible alteration of nuclear shell structure for systems with exotic proton-to-neutron ratios. Despite the important role transition strengths play in our understanding of nuclear structure, precision measurements of these quantities-generally obtained via excited state lifetime measurements - can be difficult to carry out.

While B(E2) values can be determined directly from Coulomb excitation measurements, many precision $\mathrm{B}(\mathrm{E} 2$; $0_{1}^{+} \rightarrow 2_{1}^{+}$) values are derived from lifetime measurements, where $\mathrm{B}\left(\mathrm{E} 2 ; 0_{1}^{+} \rightarrow 2_{1}^{+}\right) \propto 1 / \tau\left(2_{1}^{+}\right)$. One of the most straightforward ways to measure lifetimes is via the fasttiming electronics method.

\subsection{Fast-timing techniques}

The fast-timing technique requires two detectors with appropriately fast rise-times, standard timing electronics, and an appropriately chosen gamma cascade (e.g. the $4^{+} \rightarrow$ $2^{+} \rightarrow 0^{+}$yrast band cascade), as shown in Fig. 1(a). Gamma cascade selection will be explained in further detail below. Ignoring background subtraction, the fasttiming procedure is as follows: (1) place an energy gate on the $4^{+} \rightarrow 2^{+}$transition in one detector and the $2^{+} \rightarrow 0^{+}$

\footnotetext{
a Present address: Department of Nuclear Physics, The Australian National University, Canberra, ACT 0200 Australia; email: elizabeth.williams@anu.edu.au
}
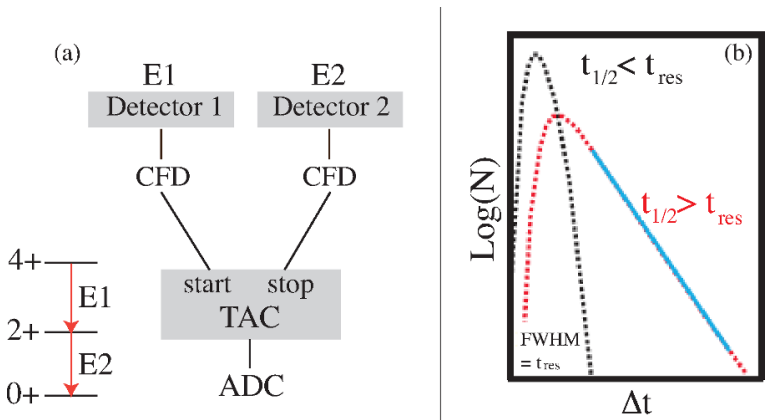

Fig. 1. (a) An illustration of the fast-timing electronics setup for one detector pair. Only the timing branch of the detector signals is shown here. (b) Schematic picture of a log plot of two possible time difference spectra obtained using the fast-timing technique. If the lifetime of the intermediate state is faster than the detectors and electronics are capable of resolving, then the (black) peak, corresponding to a shifted prompt peak or a prompt peak with some tailing will be observed. If the lifetime is sufficiently long, the red spectrum will be observed; the lifetime is directly related to the slope of the linear portion of the plot marked in blue.

transition in the other detector, and (2) project out the time differences between each pair of coincident transitions. If the lifetime of the intermediate $\left(2^{+}\right)$state is too shortmeaning that the half-life of the $2^{+}$state is smaller than the time resolution of the detectors-one simply sees a gaussian peak, sometimes with some tailing that is not well separated from the prompt peak. If the lifetime is sufficiently long, a log plot of the time difference versus counts should reveal a linear region to either side of the prompt peak that is directly related to the $2^{+}$lifetime, as illustrated in Fig. 1(b).

As this simple sketch of the technique illustrates, there are two key detector properties that must be considered 
when planning experiments using this method: energy resolution and time resolution. If one cannot place clean energy gates on the transitions of interest, or one hopes to measure a halflife that is small compared to the time resolution of the detectors, other methods (e.g. recoil distance Doppler shift [3-8], Coulomb excitation [9, 10,6]) will have to be considered.

For decades, $\mathrm{BaF}_{2}$ detectors were the standard for fasttiming measurements using gamma-gamma coincidences. $\mathrm{BaF}_{2}$ detectors offer excellent timing properties in comparison to HPGe or NaI detectors and are much more efficient for gamma spectroscopy than fast organic scintillators due to their high- $\mathrm{Z}$ composition. However, these detectors have poor energy resolution-around $10 \%$ at 511 $\mathrm{keV}$ for a 1.5 " $\mathrm{x} 1.5$ " cylindrical detector-that renders fasttiming measurements difficult for all but the simplest nuclear level schemes.

In recent years, a new inorganic scintillator, Ceriumdoped $\mathrm{LaBr}_{3}$, has become available commercially. While $\mathrm{LaBr}_{3}$ detectors have similar timing properties to $\mathrm{BaF}_{2}$ detectors, their energy resolution is $\sim 4 \%$ at $511-\mathrm{keV}$ for a 1.5 "x 1.5 " cylindrical detector, and ranges from $3-5 \%$ for ${ }^{60} \mathrm{Co}$ energies. This improved energy resolution is due to the large light output of Cerium-doped $\mathrm{LaBr}_{3}$. In principle, this improved energy resolution should render the fast-timing technique accessible to a wider range of nuclear structure experiments.

\subsection{E2 transition strengths near mid-shell}

Ideally, experiments using new detector technologies are designed to achieve two objectives: to reveal a new detector's performance advantages and possible pitfalls in a given experimental setting, and if all goes well, to do some new physics. This second objective is the reason for this work's focus on rare earth nuclei far from closed shells.

The story begins with the Interacting Boson Model-1 (IBM-1), a simple collective model that does not take into account detailed shell structure or the difference between valence protons and neutrons. In the IBM-1, one can obtain an analytical expression for the $\mathrm{B}\left(\mathrm{E} 2 ; 0_{1}^{+} \rightarrow 2_{1}^{+}\right)$values at the SU(3) (or rotational) symmetry as a function of boson number $\left(N_{B}=\right.$ valence nucleons $\left./ 2\right)[11]$ :

$$
B\left(E 2 ; 0_{1}^{+} \rightarrow 2_{1}^{+}\right)=e_{B}^{2} N_{B}\left(2 N_{B}+3\right),
$$

where $e_{B}$ is the effective boson charge. This expression reveals that these $B(E 2)$ values should increase quadratically until reaching a maximum at mid-shell, then once again fall quadratically as one proceeds to the next shell closure.

Until a few years ago, available data showed that this simple IBM-1 model was approximately correct; known $\mathrm{B}(\mathrm{E} 2)$ values did indeed maximize at mid-shell. However, J.-Y. Zhang et al. found that for rare earth nuclei, B(E2) values saturated as a function of neutron number near midshell [12] (see Fig. 1 in [12] for an illustration of this). To explain this saturation at mid-shell, they postulated that shell effects-namely, the fact that protons and neutrons are filling different orbitals - may lead to a decrease in the wavefunction overlap (and therefore, slower increase in collectivity as a function of valence nucleon) as one approaches mid-shell. To treat this within the context of the IBM-1, an "effective" valence boson number was introduced, which took into account fractional filling of the

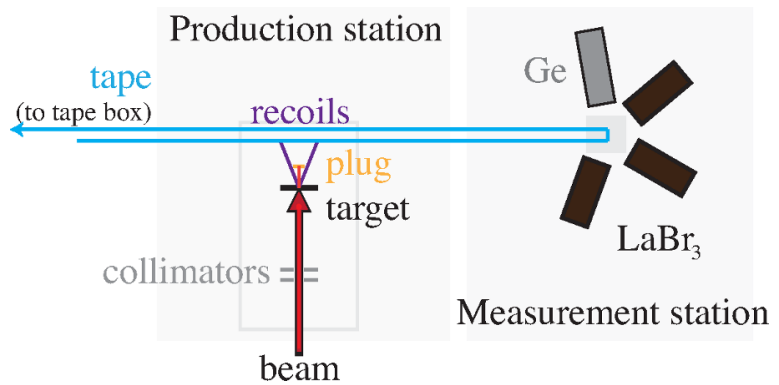

Fig. 2. Diagram of the Yale Moving Tape Collector setup. The diagram is not an accurate representation of the positions of the production and measurement stations. The target-plug separation is selected to ensure that the beam is stopped, while recoil $(\beta$ decay parent) nuclei are deposited on the tape.

shells. A microscopic approach within the projected shell model was also found [13] in the context of $g$-factor measurements, which exhibited a similar trend towards midshell.

However, a more recent measurement of E2 transition strengths in ${ }^{172} \mathrm{Hf}$ using the fast-timing technique led to a substantially revised $2_{1}^{+}$lifetime [14], prompting an experimental campaign aimed at remeasuring other $2_{1}^{+}$lifetimes in the rare earth region. The experiments on ${ }^{168} \mathrm{Hf}$ and ${ }^{174} \mathrm{~W}$ presented here were part of this campaign.

\section{Experimental setup}

To continue exploring how E2 transitions evolve towards mid-shell in rare earth nuclei, experiments measuring the $2_{1}^{+}$state lifetimes in ${ }^{168} \mathrm{Hf}$ and ${ }^{174} \mathrm{~W}$ were conducted at the Wright Nuclear Structure Laboratory at Yale University. ${ }^{168} \mathrm{Hf}$ was ideal for testing the detectors, as another highprecision lifetime measurement had already been conducted using the recoil distance doppler shift method [15]. ${ }^{174} \mathrm{~W}$ was part of a collaborative effort to remeasure E2 transitions in tungsten isotopes.

The reactions used to populate the $\beta$-decay parents of ${ }^{168} \mathrm{Hf}$ and ${ }^{174} \mathrm{~W}$ were ${ }^{159} \mathrm{~Tb}\left({ }^{16} \mathrm{O}, 7 \mathrm{n}\right){ }^{168} \mathrm{Ta}$ with a beam energy of $115 \mathrm{MeV}$ and ${ }^{169} \mathrm{Tm}\left({ }^{12} \mathrm{C}, 7 \mathrm{n}\right){ }^{174} \mathrm{Re}$ with a beam energy of $130 \mathrm{MeV}$, respectively. The beta decay products were observed using the Yale Moving Tape Collector (MTC). As shown in Fig. 2, the MTC has two stages: a production stage, where the beta-decay parents (along with other reaction channels) are produced via a heavy ion fusion reaction and deposited on a tape, and a measurement stage, where the activated tape is observed over a period of twice the half-life of the $\beta$-decay parent. In this way, one can continually produce the $\beta$-decay parents of interest while observing decay products in a low-background (off-beam) environment.

For both measurements, three 1.5"x1.5" $\mathrm{LaBr}_{3}$ detectors and one Ge detector were placed at the observation station, around the point at which the activated tape spot was transported. The electronics were set up following the logic illustrated in Fig. 1(a) for all possible permutations of the detector pairs; a maximum of six time-difference spectra were available for each lifetime measurement. The Ge detector, with its superior energy resolution, was used to verify that no other transitions would interfere with the energy gates placed on the $4^{+} \rightarrow 2^{+}$and $2^{+} \rightarrow 0^{+}$yrast tran- 

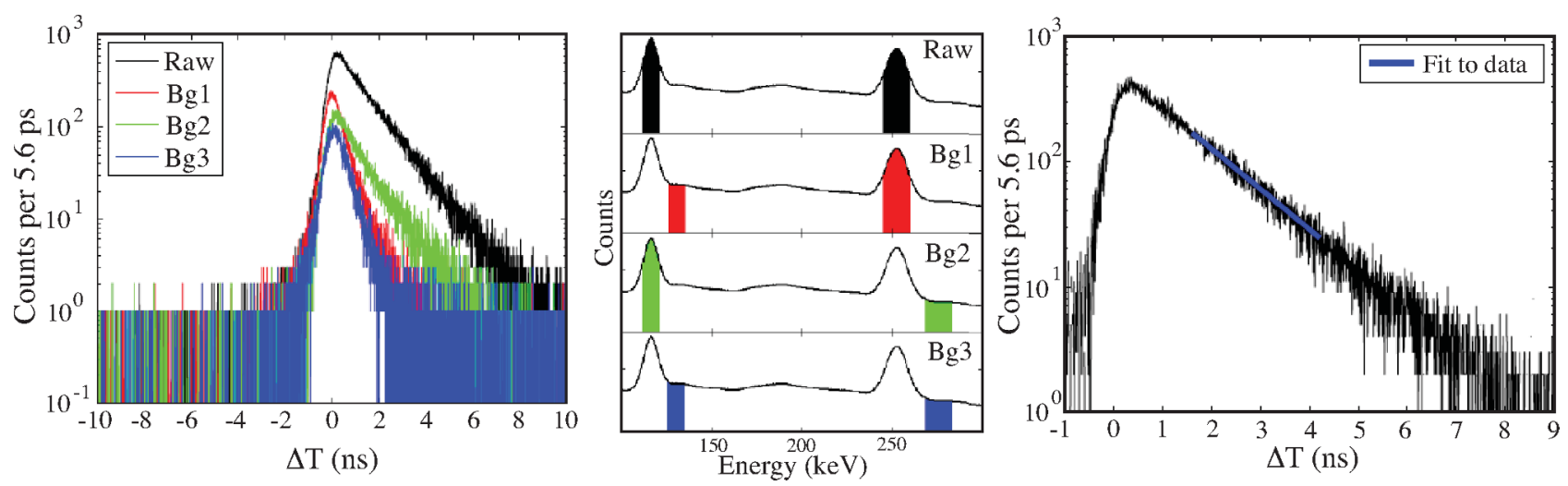

Fig. 3. The left and center panels show sample background gates and time difference spectra for the ${ }^{174} \mathrm{~W}$ experiment. The right panel shows the final background-subtracted time difference spectrum. Background gates are selected to the right of the $4^{+} \rightarrow 2^{+}$and $2^{+} \rightarrow 0^{+}$ peaks in order to avoid subtracting contributions from Compton scattered events corresponding to the transitions of interest. The excellent energy resolution of the $\mathrm{LaBr}_{3}$ detectors made this background subtraction method possible.
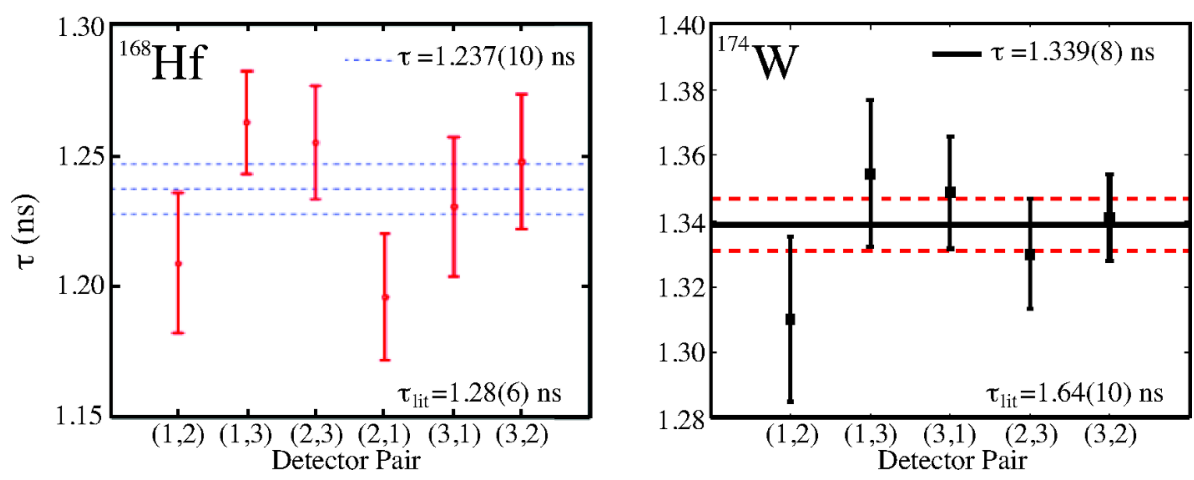

Fig. 4. Results from preliminary analyses of the $2_{1}^{+}$lifetimes for both ${ }^{168} \mathrm{Hf}$ and ${ }^{174} \mathrm{~W}$. Access to three $\mathrm{LaBr}_{3}$ detectors enhanced the precision of these measurements, resulting in an order of magnitude reduction in the statistical error when compared to literature values.

sitions of interest. All four detectors were well-shielded from the production station. Time difference spectra for all detector pairs were calibrated using cable delays. The calibrations were then checked by measuring the lifetime of the $2_{1}^{+}$state in ${ }^{152} \mathrm{Sm}$ (produced via the electron capture decay of ${ }^{152} \mathrm{Eu}$, a common calibration source).

While the method for extracting lifetimes from the $\mathrm{LaBr}_{3}$ time difference spectra has already been sketched out in Section 1.1, background subtraction is worth discussing in more detail. In all cases, the $4^{+} \rightarrow 2^{+}$and $2^{+} \rightarrow 0^{+} \gamma$ transitions will lie on top of a significant background, as one can see in the sample ${ }^{174} \mathrm{~W}$ spectra in Fig. 3(a). In order to account for this, time difference spectra for the following combinations of energy gates were obtained: (RAW) the $4^{+} \rightarrow 2^{+}$peak and the $2^{+} \rightarrow 0^{+}$peak; (BG1) the $4^{+} \rightarrow 2^{+}$peak and the region to the right of the $2^{+} \rightarrow 0^{+}$peak; (BG2) the $2^{+} \rightarrow 0^{+}$peak and the region to the right of the $4^{+} \rightarrow 2^{+}$peak; and (BG3) the regions to the right of both the $4^{+} \rightarrow 2^{+}$peak and the $2^{+} \rightarrow 0^{+}$peak. The final time difference spectrum used to extract the lifetime would be created as follows: (RAW)(BG1)-(BG2)+(BG3). This method allowed us to correct for Compton-scattered events from higher energy transitions, which we were able to identify using the Ge detector placed at the measurement station. A sample of all $\mathrm{LaBr}_{3}$ gate selections and resulting time difference projections, as well as a sample background-subtracted time-difference projection, is shown in Fig. 3.

\section{Results and Discussion}

The preliminary lifetimes for ${ }^{168} \mathrm{Hf}$ and ${ }^{174} \mathrm{~W}$, as derived from the slope of the linear portion of the backgroundsubtracted time-difference spectra, are shown for all detector pairs in Fig. 4. For ${ }^{168} \mathrm{Hf}-$ our test case- $\tau\left(2_{1}^{+}\right)$was found to be 1.237(10) ns [18], which confirms the literature value of 1.28(6) [15] and reduces the statistical error by an order of magnitude. For ${ }^{174} \mathrm{~W}, \tau\left(2_{1}^{+}\right)$was found to be $1.339(8) \mathrm{ns}$, which is significantly lower than the literature value of 1.64(10) ns [19] and again, offers a similar order of magnitude reduction in the statistical error. Estimates of systematic error for these lifetimes are $1 \%$, based on calibration data and variations in the lifetime resulting from altering prompt and background gates on the $\mathrm{LaBr}_{3}$ spectra. A full discussion of these systematic errors will be included in a future paper.

The $20 \%$ reduction in the $2_{1}^{+}$lifetime of ${ }^{174} \mathrm{~W}$ offers both a warning and a promise of new physics. It is one of several lifetime measurements in the region that have been substantially modified or improved in recent years $[14,16$, 17]. And while the overall prediction of the simple collective models - the maximum in the $\mathrm{B}\left(\mathrm{E} 2 ; 0_{1}+\rightarrow 2_{1}^{+}\right)$value at mid-shell-is correct for some rare earth isotopes, like hafnium, it is not consistent with our observations in the tungsten isotopes, as shown in Fig. 5. As these preliminary results demonstrate, it is clear only that E2 transition 

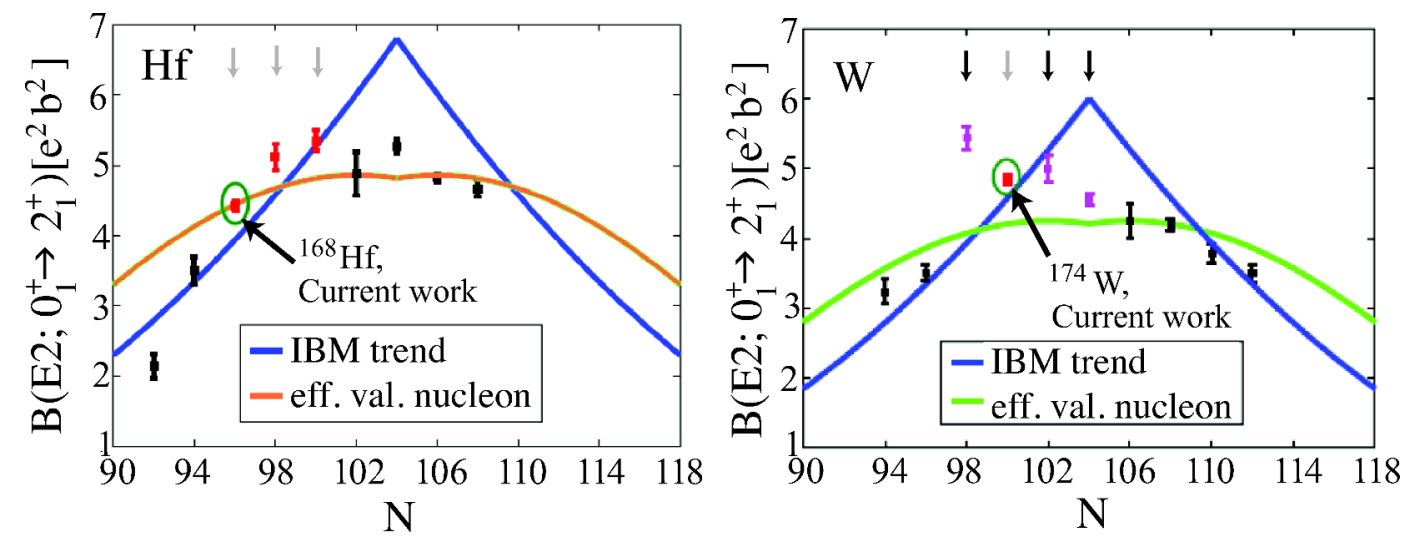

Fig. 5. Current measurements of $\mathrm{B}\left(\mathrm{E} 2 ; 0_{1}^{+} \rightarrow 2_{1}^{+}\right)$values in $\mathrm{e}^{2} \mathrm{~b}^{2}$ for hafnium (left panel) and tungsten (right panel) isotopes. The current results, in red, are circled and / or marked with gray arrows; other new measurements $[14,16,17]$ are plotted in magenta and marked with black arrows.

strengths exhibit a more intricate evolution with neutron number than the simple collective models predict.

As is typical for nuclear structure measurements, the intricacy that has been revealed by these new lifetime measurements has come with new advances in technology. The precision of these new lifetime measurements for ${ }^{168} \mathrm{Hf}$ and ${ }^{174} \mathrm{~W}$ was only achievable because of our access to multi-detector arrays and because of the improved energy resolution - and therefore, background subtraction — of the $\mathrm{LaBr}_{3}$ detectors used for this work. While the interpretation of these and similar results in rare earth nuclei are still a work in progress, these experiments have made clear how access to arrays $\mathrm{LaBr}_{3}$ detectors and other next-generation fast-timing scintillators is essential for sensitive tests of our understanding of collective nuclear structure.

\section{Acknowledgements}

This work was supported by U.S. DOE Grant No. DEFG02-91ER40609 and German DFG Grant No. Jo391/3-2.

\section{References}

1. V. Werner, N. Pietralla, P. von Brentano, R.F. Casten, R.V. Jolos, Phys. Rev. C 61, 021301(R) (2000)

2. V. Werner, E. Williams, R.J. Casperson, R.F. Casten, C. Scholl, P. von Brentano, Phys. Rev. C 78, 051303 (2008)

3. A.Z. Schwarzschild, E.K. Warburton, Ann. Rev. Nucl. Sci. 18, 265 (1968)

4. A. Dewald, S. Harissopulos, P. von Brentano, Z. Phys. A 334, 163 (1989)

5. E.A. McCutchan, N.V. Zamfir, R.F. Casten, H. Ai, H. Amro, M. Babilon, D.S. Brenner, G. Gurdal, A. Heinz, R.O. Hughes et al., Phys. Rev. C 73, 034303 (2006)

6. E. Williams, C. Plettner, E.A. McCutchan, H. Levine, N.V. Zamfir, R.B. Cakirli, R.F. Casten, H. Ai, C.W. Beausang, G. Gurdal et al., Phys. Rev. C 74, 024302 (2006)

7. R. Lüttke, E.A. McCutchan, V. Werner, K. Alexandrova, R.F. Casten, A. Heinz, A.F. Mertz, J. Qian,
B. Shoraka, J.R. Terry et al., Phys. Rev. C 85, 017301 (2012)

8. D. Radeck, V. Werner, G. Ilie, N. Cooper, V. Anagnostatou, T. Ahn, L. Bettermann, R.J. Casperson, R. Chevrier, A. Heinz et al., Phys. Rev. C 85, 014301 (2012)

9. K. Alder, A. Winther, eds., Coulomb Excitation (Academic Press, 1966)

10. D. Cline, Ann. Rev. Nucl. Part. Sci. 36, 683 (1986)

11. F. Iachello, A. Arima, The Interacting Boson Model (Cambridge University Press, Cambridge, United Kingdom, 1987)

12. J.Y. Zhang, R.F. Casten, A. Wolf, Z. Berant, R.B. Cakirli, N.V. Zamfir, E.A. McCutchan, Phys. Rev. C 73, 037301 (2006)

13. B.A. Bian, Y.M. Di, G.L. Long, Y. Sun, J.Y. Zhang, J.A. Sheikh, Phys. Rev. C 75, 014312 (2007)

14. V. Werner, J.R. Terry, M. Bunce, Z. Berant, J. Phys: Conf. Series 205, 012025 (2009)

15. A. Costin, M. Reese, H. Ai, R.F. Casten, K. Dusling, C.R. Fitzpatrick, G. Gürdal, A. Heinz, E.A. McCutchan, D.A. Meyer et al., Phys. Rev. C 79, 024307 (2009)

16. J.M. Régis, T. Materna, S. Christen, C. Bernards, N. Braun, G. Breuer, C. Fransen, S. Heinze, J. Jolie, T. Meersschaut et al., Nucl. Instr. and Meth. A 606, 466 (2009)

17. M. Rudigier, J.M. Régis, J. Jolie, K.O. Zell, C. Fransen, Nucl. Phys. A 847, 89 (2010)

18. A. Wolf, Z. Berant, V. Werner, A. Heinz, R.F. Casten, R.J. Casperson, D.S. Brenner, R. Chevrier, D. McCarthy, G. Ilie et al., Phys. Rev. C 85, 037304 (2012)

19. J. Gascon, P. Taras, P.V. Esbroek, H.R. Andrews, D.C. Radford, D. Ward, A. Christy, Nucl. Phys. A472, 558 (1987) 